\title{
O Programa Nacional de Controle do Tabagismo no município do Rio de Janeiro: ligações que promovem oportunidades
}

\section{The National Smoking Control Program in Rio de Janeiro district: telephone calls that promotes opportunities}

\author{
Paulo Cesar Moreira Campos' (D), Diego Viegas Sato Barbosa', Marcia Gomide² \\ 'Grupo de Pesquisa e Documentação sobre Empresariamento na Saúde (GPDES), Instituto de Estudos de Saúde Coletiva (IESC), \\ Universidade Federal do Rio de Janeiro (UFRJ) - Rio de Janeiro, RJ, Brasil. \\ 2Instituto de Estudos de Saúde Coletiva (IESC), Universidade Federal do Rio de Janeiro (UFRJ)-Rio de Janeiro, RJ, Brasil.
}

\begin{abstract}
Como citar: Campos PCM, Barbosa DVS, Gomide M. O Programa Nacional de Controle do Tabagismo no município do Rio de Janeiro: ligações que promovem oportunidades. Cad Saúde Colet, 2021; Ahead of Print. https://doi.org/10.1590/1414462X202129030206
\end{abstract}

\section{Resumo}

Introdução: Apesar de o Programa Nacional de Controle do Tabagismo (PNCT) ser um dos mais efetivos da América Latina, resultando em contínua queda do hábito de fumar até 2013, sua prevalência vem se mostrando importante em algumas regiões do Brasil.Objetivo: Analisar a situação da rede do PNCT no município do Rio de Janeiro no ano de 2015 em relação à captação de tabagistas sob a óptica da análise de redes sociais.

Método: A pesquisa possui caráter exploratório-descritivo e qualitativo. As informações foram obtidas por meio de ligações telefônicas, realizadas a todas as unidades de saúde participantes do PNCT, totalizando 183 ligações. Para o complemento da análise à abordagem pela análise de redes sociais, foi utilizada a metodologia conhecida como matriz FOFA (Forças, Oportunidades, Fraquezas e Ameaças).

Resultados: Do total de 183 unidades, foi obtida resposta de 68. Apesar de mais da metade dos atendimentos ter ocorrido por encaminhamento dos profissionais de saúde, quantidade significativa se deu por meios informais.

Conclusão: Conclui-se que existem diversos fatores relacionados a forças, oportunidades, fraquezas e ameaças. Porém, se pouco falta em relação a recursos e estratégias para a melhoria da captação, muito há a fazer para que as ligações telefônicas sejam oportunidades de mudança.

Palavras-chave: tabagismo; avaliação; programas de saúde; políticas de saúde.

\section{ABSTRACT}

Background: Although the National Smoking Control Program - PNCT is one of the most effective in Latin America, resulting in a continuous decline in the prevalence of smoking until 2013, its prevalence has been shown to be important in some regions of Brazil.

Objective: To analyze the situation of the PNCT network in the Municipality of Rio de Janeiro in 2015, regarding the uptake of smokers from the perspective of Social Network Analysis.

Method: This is an exploratory-descriptive and qualitative study. Information was obtained through telephone calls made to all Health Units participating in the PNCT, totaling 183 calls. To complement the analysis of the approach through the analysis of social networks, we used the methodology known as SWOT matrix - strengths, opportunities, weaknesses, and threats.

Este é um artigo publicado em acesso aberto (Open Access) sob a licença Creative Commons Attribution, que permite uso, distribuição e reprodução em qualquer meio, sem restrições desde que o trabalho original seja corretamente citado.
Trabalho realizado no município do Rio de Janeiro (RJ), Brasil.

Correspondência: Paulo Cesar Moreira Campos. E-mail: paulotorreao.pt@gmail.com

Fonte de financiamento: nenhuma.

Conflito de interesse: nada a declarar

Recebido em: Jun. 06, 2019. Aprovado em: Abr. 05, 2020 
Results: From a total of 183 units, 68 responded. Although more than half of the consultations took place through referrals from health professionals, a significant number took place through informal means. Conclusion: It is concluded that several factors related to weaknesses, strengths, opportunities, and threats exist. However, if there is little lack of resources and strategies to improve uptake, there is much to be done so that telephone calls are opportunities for change.

Keywords: smoking; evaluation; health programs; health policies.

\section{INTRODUÇÃO}

Em 1985, o Ministério da Saúde lançou o Programa Nacional de Controle do Tabagismo (PNCT) com o objetivo de prevenir o início da utilização de derivados do tabaco e estimular o abandono do fumo entre os já dependentes. Apesar de o PNCT ser um dos mais efetivos da América Latina, resultando em contínua queda da prevalência tabagista até ano de $2013 \mathrm{e}$ apontando um percentual total de adultos fumantes de $14,7 \%$, sua prevalência volta a ser preocupante ${ }^{1}$ Segundo a Pesquisa Nacional de Saúde (PNS)², o tabagismo vem se mostrando importante em algumas regiões e entre populações em estado de fragilidade social2,3.

Neste contexto, o Ministério da Saúde publicou, em 5 de abril de 2013, a Portaria no 571, que atualiza as diretrizes de cuidado à pessoa tabagista no âmbito da Rede de Atenção à Saúde das Pessoas com Doenças Crônicas do Sistema Único de Saúde ${ }^{4}$, propondo novas orientações sobre a adesão ao PNCT, a programação para a aquisição da medicação e o estabelecimento de responsabilidades.

As estratégias adotadas na cidade do Rio de Janeiro para o controle do uso de tabaco se baseiam em tais diretrizes. Segundo a Secretaria Municipal de Saúde desse município ${ }^{5}$ o PNCT segue cinco linhas de ação: (1) ambientes $100 \%$ livres de fumo; (2) tratamento para deixar de fumar; (3) prevenção da iniciação no tabagismo; (4) mobilização em datas comemorativas; e (5) divulgação da legislação. Entre os serviços prestados pela Secretaria Municipal de Saúde do Rio de Janeiro ${ }^{5}$ estão medidas preventivas e curativas, propostas como abordagem integral, com tratamento para fumantes e capacitação de profissionais.

Além da incorporação de tais diretrizes, em obediência aos princípios do Sistema Único de Saúde (equidade, integralidade e acesso universal), é imprescindível haver descentralização e implementação dessas políticas. Para tal, a rede de atendimento precisa exercer papel ativo, captando e conectando usuários aos serviços pertinentes.

Uma rede de serviço estruturada, conforme preconizado pelo Ministério da Saúde, promove acesso rápido ao apoio social, importante alicerce a programas ${ }^{6}$, notadamente para o tabagista. Assim, a intenção aqui é analisar a situação da rede do PNCT no município do Rio de Janeiro em relação à captação do tabagista.

\section{MÉTODO}

Trata-se de uma pesquisa descritiva e exploratória, conjugando a perspectiva teórica da análise de redes sociais (ARS) com o método da matriz de avaliação FOFA. Na ARS, são descritas relações sociais condicionantes das escolhas dos indivíduos com a estrutura social a que pertencem ${ }^{7}$, propiciando identificar os processos relacionais ${ }^{8}$ tais como acesso a oportunidades e pressões ${ }^{9}$. Já a matriz FOFA analisa as Forças (F), Oportunidades (O), Fraquezas, (F) e Ameaças (A), verificadas no contexto considerado ${ }^{10}$. Seu âmago é indicar os fatores essenciais a dada reorganização"

Por ser uma pesquisa de caráter exploratório-descritivo, é limitada pelo fato de seus resultados demonstrarem a situação da amostra no período de execução, bem como não generalização para outras populações.

Compuseram o escopo da pesquisa as unidades de saúde que ofertavam o PNCT no município do Rio de Janeiro. A coleta de dados se dividiu em duas etapas: (1) busca por dados de livre acesso e (2) informações obtidas diretamente de profissionais das unidades de saúde, nos moldes de "inquérito telefônico", realizado pela Secretaria de Vigilância em Saúde do Ministério da Saúde, denominado VIGITEL². Esses telefones foram obtidos das listas de unidades, disponíveis no site da Secretaria Municipal de Saúde ${ }^{5}$, totalizando 183 unidades de 
atendimento aos tabagistas registradas no Programa. Foi feita a opção por utilizar as ligações telefônicas para não só viabilizar o contato com todas as unidades, mas também pelo fato de as ligações representarem oportunidades de mudança para o tabagista (Tabela 1).

Tabela 1. Unidades participantes do Programa Nacional de Controle do Tabagismo (PNCT)

\begin{tabular}{cc}
\hline Unidades na primeira lista & 196 \\
\hline Entraram no PNCT na segunda lista & 16 \\
\hline Saíram do PNCT na segunda lista & 29 \\
\hline Unidades na segunda lista & 183
\end{tabular}

O procedimento de ligações telefônicas foi realizado durante três meses, das 10 horas às 20 horas. Para cada número, foi feito um telefonema inicial. Caso a ligação não fosse atendida ou o número estivesse ocupado, eram feitas mais duas tentativas, com intervalo de 2 minutos entre elas. Não conseguind o realizar o contato, o número era reservado para posterior operação de religação, seguindo o mesmo procedimento. Em unidades com mais de uma linha telefônica, o procedimento foi repetido, conforme descrito, para cada número.

As indagações foram dirigidas a quem atendesse à chamada, sendo de cunho informativo, nos moldes requeridos por qualquer cidadão potencialmente usuário do Programa, abordando temas como a forma de adesão, o funcionamento do Programa, e a possibilidade de transferência para outras unidades (encaminhamento de pacientes). Nenhuma identificação pessoal foi solicitada aos profissionais que atendiam às ligações. As informações obtidas forneceram elementos de comparação entre a realidade do PNCT e os procedimentos preconizados, bem como a verificação do grau de dificuldade enfrentado pelos usuários na obtenção de informações básicas ao telefone sobre sua adesão. A pesquisa foi aprovada pelo Comitê de Ética em Pesquisa do Instituto de Estudos em Saúde Coletiva da Universidade Federal do Rio de Janeiro (UFRJ), Parecer n² 2.295.368.

\section{RESULTADOSE DISCUSSÃO}

Das 183 unidades cadastradas, foi obtida resposta às chamadas de apenas 68 . Das 115 unidades restantes, 3, apesar de constarem na lista, não tinham telefones. Das demais 112, em 49 o sinal soou até o desligamento automático da chamada e em 56 o sinal estava sempre de ocupado. Também não foi possível completar a ligação em 2 casos. Por fim, houve situações em que o telefone era residencial ( 2 casos), empresarial ( 2 casos) ou estava desativado (1 caso) (Tabela 2).

Tabela 2. Contatos telefônicos exitosos

\begin{tabular}{cc}
\hline Efetuados & 68 \\
\hline Não Efetuados & 115 \\
\hline
\end{tabular}

É preocupante o fato de não se ter obtido êxito em mais da metade das ligações. Essa dificuldade se reverte em uma barreira a mais para o tabagista. A função da lista de unidades do PNCT é a de fornecer o pronto acesso ao tratamento. Números de telefones errados e dificuldade 
de obter informações, em importante momento decisório para o tabagista, são perdas de oportunidades preciosas, mesmo considerando outras formas de chegada ao Programa.

Nas 68 ligações bem-sucedidas, foi verificado, de modo não excludente, que em 16 unidades os tabagistas chegavam, principalmente, por demanda espontânea; em 18, por intermédio das consultas; e em 25, por agentes comunitários de saúde (ACS). Em outras 4 unidades, os tabagistas chegavam, sobretudo, por informação dos próprios funcionários da unidade, e em 22, principalmente pela informalidade do "boca a boca". Mesmo que chegassem por mais de uma forma, a maneira preponderante foi a da informalidade da rede espontânea ("boca a boca") entre pessoas que se conheciam e compartilhavam suas experiências. Esse resultado, mesmo considerando as limitações desta pesquisa, corrobora a importância das redes sociais no acesso a informações e serviços de saúde ${ }^{12}$ a partir de ligações espontâneas entre os indivíduos ${ }^{13}$, o que facilita, sobremaneira, a atuação das redes de apoio, visto que estas mobilizam uma diversidade de auxílios ${ }^{14}$, tal como informações. $O$ acesso à informação pode ser entendido como um recurso comunitário formado pelas redes de relações ${ }^{7}$, importantes para a afluência de tabagistas.

Como ações para atrair o tabagista, foram obtidas informações quanto à realização de campanhas, veiculação de propagandas, folhetos, cartazes e ações de busca ativa diversificadas, tais como articulações com centros, líderes comunitários, igrejas, eventos, convites, grupos de caminhada e visitas domiciliares. Todas essas estratégias foram apontadas em números diversos e esparsos entre as unidades, sem haver um padrão de captação e acolhimento ao tabagista entre elas, mas demonstrando potencial criativo.

Da mesma forma, não foi verificado, a partir das informações prestadas, um protocolo de encaminhamento, nem para a manutenção ou não dos casos não adstritos. Das 68 unidades, 38 não realizavam encaminhamentos, segundo o informado, sendo que a maioria dizia indicar aos pacientes que buscassem, eles mesmos, a unidade de referência. Poucas aguardavam contato da equipe de unidade para enviar a ficha do paciente ou sugerir novo local.

Apesar de o encaminhamento de fumantes para a realização do Programa em outra unidade ser o preconizado e, em certos casos, o desejável, paradoxalmente tal procedimento pode ser uma ameaça à eficácia do Programa. O tabagista tem natureza recalcitrante, perdendo o encorajamento e a motivação em qualquer dificuldade. Por isso, o Programa deve minimizar todo elemento que dificulte o pronto acesso dos tabagistas ao tratamento.

Importa considerar o quão crucial é o momento do encaminhamento e o quanto houve de desatenção à prestação de tal indicação. As informaçães obtidas a esse respeito não foram encorajadoras, já que: (a) indicaram a busca pelo site "Onde Ser Atendido"; (b) disseram que existia uma unidade próxima da casa do paciente e davam o endereço; e (c) explicaram ao fumante que não era possível fazer o encaminhamento. Fica nítida a perda de oportunidades de captação em momento tão decisivo ao fumante recalcitrante.

Para agravar a sugestão do encaminhamento a partir do site "Onde Ser Atendido", as unidades ali listadas não coincidiam com as da lista do próprio PNCT. Além de resultar em perda de adesão, isso pode levar pacientes a unidades que não participem do PNCT, atrasando ou até interrompendo o acesso dos tabagistas ao Programa. Ser encaminhado para uma unidade que não realiza o tratamento pode levar o fumante à frustração e à desistência.

O preconizado pelo Ministério da Saúde é que o tratamento para a cessação do tabagismo seja oferecido em redes de cooperação e parcerias ${ }^{1}$. Na prática, isso não parecia ocorrer. O resultado dessa desconexão é um empobrecimento do fluxo de informações, dificultando a implantação de conduta padrão do Sistema Único de Saúde ${ }^{15}$. Como uma das diretrizes do PNCT é aumentar o acesso do fumante ao tratamento, a rede de apoio terapêutico deve estar em conexão para viabilizar a cessação do hábito de fumar. Essa ausência de cooperação pode causar a perda de adesão.

Se, por um lado, a ausência de uma estratégia de cooperação em rede pode estar comprometendo a captação de fumantes para o Programa, por outro a busca ativa pelos ACS parece ser uma forma relevante de captação do tabagista. Esse contexto consolida a importância da associação entre os serviços de Atenção Primária à Saúde e a comunidade². Sendo o ACS membro da comunidade, por vezes ele pode fazer parte da rede social e das redes de apoio de 
alguns tabagistas. Assim, centralizado na rede, ele tem condições de identificar situações de risco individual, encaminhar pessoas, acompanhar sua situação de saúde e ajudá-lo a conseguir os resultados esperados, como preconizado nas políticas de saúde².

Por conseguinte, ao ser realizado o tratamento do tabagista, deve ser levada em consideração a disponibilidade de apoio emocional fornecido a partir de sua rede pessoal, conforme já vem sendo apontado desde fins do século XX, visando aumentar a possibilidade de sucesso do Programa ${ }^{16}$. Nesse contexto, é mais do que urgente incluir os profissionais de saúde na escala de altos valores, como elementos preponderantes nesse processo, notadamente durante as consultas.

As consultas com os profissionais de saúde foram mencionadas por 18 funcionários como a forma pela qual os fumantes tomaram conhecimento do Programa, sendo uma relevante oportunidade para que os profissionais abordassem a questão com os pacientes ${ }^{4}$. Elas poderiam estimular a participação no tratamento com a inclusão imediata no Programa e incentivar que os profissionais de saúde questionassem, mesmo os mais jovens, sobre o hábito de fumar, realizando o imediato encaminhamento, em caso de resposta positiva, e reforçando o desestímulo, em caso negativo. Isso porque o número de casos de tabagismo vem aumentando entre os jovens, sobretudo na região Sudeste ${ }^{1}$, requerendo investimento para mais visibilidade ao tema e modernizando estratégias para atingir esse público.

Poucas unidades pareceram utilizar meios de comunicação atuais e datas comemorativas como momento sensibilizador. Entre os serviços que deveriam ser prestados estavam justamente o de apoio e divulgação de ações educativas nas unidades de saúde e outras instituições em datas comemorativas ${ }^{5}$

Apesar da ampla cobertura, incluindo por vezes a existência de mais de uma unidade em bairros de maior extensão territorial, não pareceu haver contato entre elas que viesse a sugerir a existência de uma estratégia de ação em rede. Nesse sentido, a atualização da lista de telefones das unidades com o PNCT deveria ser encarada com maior rigor. Testemunho disso é que ela foi referida somente uma vez, embora a menção da demanda espontânea em 16 ligações não exclua a possibilidade de que esses fumantes tenham chegado às unidades a partir de consulta à lista. Logo, não é seguro considerar que a lista seja pouco consultada pelo tabagista, apesar dos sérios problemas identificados. Isso provavelmente não teria ocorrido se houvesse troca de informações entre as unidades e a coordenação do PNCT, à guisa de uma organização em rede ${ }^{17}$.

Como redes de apoio, complementando as premissas do PNCT, as unidades poderiam utilizar áreas externas, como igrejas, escolas e sedes de associação de moradores, locais já aproveitados na divulgação do Programa por algumas unidades. Contudo, seria necessário que houvesse o preparo e a sensibilização dos profissionais para tal, mas sendo considerados os territórios.

A "territorialização" de iniciativas do Sistema Único de Saúde, como a Estratégia Saúde da Família, é a recomendada ${ }^{18}$. Entretanto, o acesso ao Programa, restrito a residentes da área de abrangência da unidade, pode representar, no caso do tabagista, um empecilho ao tratamento. Talvez por estarem cientes dessa condição diferenciada, 16 unidades indicaram assim proceder.

Mais uma vez, se estivesse o PNCT organizado em rede, ele poderia receber o paciente, inscrevê-lo fora de sua região adstrita e, em seguida, encaminhá-lo para realizar o tratamento em unidade próxima à sua residência, seguindo, assim, a normatização territorial. Ou, nesse caso, essa premissa não seria aplicada de fato. Por exemplo, o fumante poderia optar por realizar o tratamento em unidade perto de seu trabalho ou de sua residência, em função da maior disponibilidade de tempo para realizá-lo. O planejamento da disponibilização do tratamento precisaria acompanhar a lógica. Entretanto, o tabagismo não é necessariamente vinculado a condições de regiões adstritas numérica e geograficamente como as doenças infecciosas; é uma doença de contágio social, exigindo uma abordagem inovadora, que considere suas relações sociais ${ }^{19}$

Esse procedimento já ocorria em muitas unidades, as quais os encaminhavam somente quando não podiam participar do Programa por motivos diversos (9 casos). Entretanto, existem informações contraditórias. Algumas unidades afirmavam receber orientação da coordenação 
do Programa para atender todo tabagista independentemente de seu local de residência. Outras aceitavam adesão somente dos residentes da área adstrita ${ }^{5}$.

A atuação em rede e a valorização da rede pessoal do fumante são quesitos, muito provavelmente, preponderantes para a adesão ao PNCT. Uma rede social está em constante construção ${ }^{9}$ e essa condição deveria ser levada em consideração na estruturação das redes de apoio ao tabagista, o que estaria de acordo com as diretrizes do $\mathrm{PNCT}^{4}$. Além disso, a rede pessoal é tão importante quanto a rede de apoio organizada pelo Programa ${ }^{6}$ já que funcionaria como complementar e incentivadora do tratamento, resultando em maior possibilidade de êxito.

As relações sociais provenientes da rede social em que o indivíduo está inserido estabelecem um tecido condicionante de suas ações ${ }^{7}$, as quais podem gerar influências comportamentais de uns sobre os de outros ${ }^{12}$. Além disso, em consequência de pertencer a uma rede social, benefícios são assegurados ${ }^{20}$. $\mathrm{O}$ apoio social fornece informação e/ou auxílio material procedente de grupos ou pessoas próximas, que resultam em comportamentos positivo ${ }^{20}$. Essas seriam condições altamente desejáveis em um cenário de estímulo à decisão de cessar o tabagismo e de aderência ao Programa.

Atingir tais condições pode ser mais viável do que o presumível. A análise do Programa sob a perspectiva do método FOFA (Tabela 3) evidenciou que as forças do PNCT pareciam relacionar-se à descentralização do atendimento, com um número grande de unidades que ofertavam o tratamento, em consonância com o preconizado 4 . Promissor também foi o papel das redes espontâneas, que se mostraram ser de suma importância na promoção e captação de pacientes, bem como na realização do tratamento.

Tabela 3. Matriz FOFA - Forças (F), Oportunidades (O), Fraquezas, (F) e Ameaças (A)

\begin{tabular}{cc}
\hline FORÇAS (internas) & OPORTUNIDADES (externas) \\
\hline Horizontalização do Programa & $\begin{array}{c}\text { Papel da rede espontânea na promoção do } \\
\text { Programa }\end{array}$ \\
\hline Agente comunitário de saúde & Lista do PNCT \\
\hline Grande número de unidades participantes do & Site da Secretaria de Saúde \\
\hline Participação da clínica da família & Parcerias \\
\hline Núcleos de Apoio à Saúde da Família (Nasf) & Uso de centros comunitários \\
\hline FRAQUEZAS (internas) & AMEAÇAS (externas) \\
\hline Não existência de uma normatização & Lista desatualizada do PNCT \\
\hline Ausência de diálogo & Excesso de demanda x falta de paciente \\
\hline Confusão no encaminhamento de tabagistas para & Descredenciamento \\
\hline Dificuldade em promover o Programa & Bairros sem unidades que realizam o Programa \\
\hline Não funcionamento em rede. & Falta de insumos \\
\hline
\end{tabular}

Outra força do Programa foi a dedicação dos profissionais que o executavam, resultando no grande número de unidades participantes do PNCT que recebiam tabagistas de áreas distintas da adstrita (50 em 68 das informações), o que, mesmo sendo contrário ao preconizado, sob o aspecto recalcitrante do tabagista, era procedimento positivo, que poderia ser normatizado, confirmando a tese de que são os comportamentos que ditam as normas vigentes ${ }^{9}$. 
Considerada uma oportunidade, a horizontalidade do Programa se adequava às diversas realidades e, mesmo resultando em ausência de conduta padrão, favorecia a adesão ao Programa. Outras oportunidades observadas foram procedimentos que levavam ao aproveitamento dos recursos humanos e materiais disponíveis em cada unidade, por exemplo, profissionais com formação em acupuntura ou em educação física, materiais como filmes e folhetos explicativos, datashow etc. Tornar o aproveitamento desses recursos uma orientação a ser adotada levaria a um procedimento comum integrante do planejamento estratégico do PNCT.

Como fraquezas, foi verificada uma série de unidades de saúde que realizavam o Programa sem haver vínculos, sem se organizarem em redes de apoio de fato. Tal isolacionismo é um equívoco. O tabagismo é uma doença crônica e de contágio social, com graves repercussões sobre a saúde, cujos pacientes, por vezes, já apresentam sequelas quando buscam tratamento - necessitando urgentemente de ingresso no Programa -, além de estar frequentemente associado a distúrbios psiquiátricos ${ }^{21,22}$.

A atuação das unidades deve ser regida por uma sistematização que estimule a rede de relações com um objetivo comum ${ }^{17}$. Não a rede de relações pessoais, pois esta é invisível e espontânea, não sendo sinônimo de rede de apoio ${ }^{19}$. Contudo, esta última é facilitada pela primeira. A rede de apoio é caracterizada por um grupo de pessoas com condições de fornecer auxílio e apoio a um indivíduo ou grupo familiar23.

As ameaças ao Programa aparentam ser consequência da ausência de ações compartilhadas e complementares. Por exemplo, o descredenciamento e a ausência de unidades em alguns bairros, o que pode resultar em excesso de demanda nas remanescentes, poderiam ser evitados se atuassem em rede. Seria útil instituir diretrizes para a organização das unidades do PNCT em rede ${ }^{11}$. Dessa forma, irregularidades que levassem a possíveis insucessos na abordagem e tratamento dos tabagistas poderiam ser suprimidas pela facilitação resultante das ações conjuntas ${ }^{17}$. Uma questão importante foi a dificuldade de contatar as unidades do PNCT por telefone. Essa condição compromete não só a adesão ao tratamento, mas também a comunicação entre elas. Embora deva haver outra forma de se comunicar, o contato telefônico apresenta-se como a mais direta, viabilizando a discussão e a troca de informações sobre pacientes e serviços. Ademais, o fato de a lista do PNCT não estar atualizada sugere a falta de seu uso pelos profissionais.

Em síntese, as oportunidades de avanço para o Programa são menos complexas e dispendiosas do que se poderia supor, visto que as forças para tal já estão instituídas e consolidadas no Programa. As oportunidades se fazem pela própria tendência humana - nesse caso, no perfil do tabagista - de buscar por integração e aceitação. E as redes espontâneas comprovaram tal natureza. Suas fraquezas não são de difícil superação, já que parecem proceder apenas da falta de interação e diálogo retroalimentados pela ausência de conduta padronizada, mas são ameaças insidiosas que carecem de coibição tão urgente e de simples resolução.

Nota-se, portanto, que, apesar de o PCNT no município do Rio de Janeiro estar amplamente distribuído, não há garantia de adesão ao tratamento. É preciso considerar as características extremamente particulares dessa doença, sujeita ao instante decisório do indivíduo, assim como o acesso preconizado nas diretrizes programáticas. A boa conclusão é que o esperado pode ser alcançado sem onerar custos, já que reclama apenas o estímulo à interlocução entre programas, nos programas, entre pessoas e com pessoas, por ligações que transformam.

\section{CONSIDERAÇÕES FINAIS}

Pelas informações obtidas por meio das chamadas telefônicas, foi percebida ausência de uma padronização do modus operandi entre as unidades participantes do PNCT no município do Rio de Janeiro. Apesar do preconizado, não existia uma estrutura em rede, o que poderia levar a uma redução na captação e no tratamento dos tabagistas. Também não pareceu ser do domínio de todos os profissionais a existência de diretrizes para o encaminhamento dos fumantes.

Um dos achados positivos foi o compartilhamento de unidades básicas de saúde pela Estratégia Saúde da Família e PNCT, o que facilitava a captação de tabagistas pelos ACS. Outro 
ponto digno de nota foram as consultas e campanhas nas clínicas da família, importantes elementos na detecção e inclusão de fumantes no Programa. $O$ acesso também foi favorecido pela ação das redes espontâneas dos tabagistas, componente relevante para a inclusão deles no Programa.

Dessa forma, sem custos elevados ou modificações operacionais drásticas, o PNCT do Rio de Janeiro tem possibilidades amplas de voltar a controlar o tabagismo. As perspectivas são boas, bastando incentivar ligações que transformam.

\section{REFERÊNCIAS}

1. Brasil. Ministério da Saúde. Portaria n 571, de 5 de Abril de 2013. Atualiza as diretrizes de cuidado à pessoa tabagista no âmbito da Rede de Atenção à Saúde das Pessoas com Doenças Crônicas do Sistema Único de Saúde (SUS) e dá outras providências. Diário Oficial da União [Internet], Brasília, 2013 [citado em 27 Jun 2015]. Disponível em: http://bvsms.saude.gov.br/bvs/saudelegis/gm/2013/prt0571_05_04_2013. html

2. Instituto Nacional do Câncer José Alencar Gomes da Silva. Observatório da política Nacional do controle do tabaco [Internet]. Rio de Janeiro: INCA; 2021. Disponível em: https://www.inca.gov.br/observatorioda-politica-nacional-de-controle-do-tabaco/dados-e-numeros-prevalencia-tabagismo.

3. Instituto Nacional do Câncer José Alencar Gomes da Silva. Comissão Nacional para Implementação da Convenção-Quadro para controle do Tabaco (CONICQ). Política Nacional de controle do tabaco: relatório de gestão e progresso 2011-2012. Rio de Janeiro: INCA; 2014. 132 p.

4. Instituto Nacional do Câncer José Alencar Gomes da Silva. Programa Nacional de Controle do Tabagismo (PNCT) Tratamento do Tabagismo [Internet]. Rio de Janeiro: INCA; 2021. Disponível em: https://www.inca. gov.br/observatorio-da-politica-nacional-de-controle-do-tabaco.

5. Rio de Janeiro. Prefeitura Municipal. Secretaria Municipal de Saúde. Programa de Controle do Tabagismo [Internet]. Rio de Janeiro: Prefeitura Municipal; 2015 [acesso em 15 Maio 2015]. Disponível em: http:// www.rio.rj.gov.br/web/sms/tabagismo

6. Gomide M, Costa PHA, Colugnati FAB. A análise de redes sociais nas redes de atenção aos usuários de álcool e outras drogas. In: Ronzani TM, Costa PHA, Mota DCB, Laport TJ, organizadores. Redes de atenção aos usuários de drogas: políticas e práticas. São Paulo: Cortez; 2015. p. 129-54

7. Marteleto RM, Silva ABO. Redes e capital social: o enfoque da informação para o desenvolvimento local. Ci Inf. 2004;33(3):41-9.

8. Sant'Ana LF. Papel do servidor público na interação entre Estado e sociedade: uma análise sob o prisma de redes. [dissertação]. Belo Horizonte: Programa de Pós-Graduação em Comunicação Social, Pontifícia Universidade Católica de Minas Gerais; 2011.

9. Degenne A, Forse M. Les Réseaux Sociaux. Paris: Armand Colin; 2004. 295 p.

10. Anjasni B. Swot assesment of the community potency to determine the strategic planning for volcano eruption disaster management (Case study in Cangkringan, Yogyakarta province). Procedia Environ Sci. 2013;17:337-43. http://dx.doi.org/10.1016/j.proenv.2013.02.046.

11. Rachid G, El Fadel M. Comparative swot analysis of strategic environmental assessement systems in the Middle East and North Africa region. J Environ Manage. 2013;125(15):85-93. http://dx.doi.org/10.1016/j. jenvman.2013.03.053. PMid:23648267.

12. Eriksson M. Social capital and health implications for health promotion. Glob Health Action. 2011;4(1):5611. http://dx.doi.org/10.3402/gha.v4i0.5611. PMid:21311607.

13. Grossetti M. L'imprévisibilité dans les parcours sociaux. Cah Int Sociol. 2006;1(120):5-28. http://dx.doi. org/10.3917/cis.120.0005.

14. Marques E, Bichir R. Redes de Apoio Social no Rio de Janeiro e em São Paulo. Novos Estudos. 2011;65-83.

15. Mattos RA. Os sentidos da integralidade: algumas reflexões acerca de valores que merecem ser definidos. In: Pinheiro R, Mattos RA, editors. Os sentidos da integralidade na atenção e no cuidado à saúde. Rio de Janeiro: UERJ; 2001. p. 43-68.

16. Ostergren PO, Hanson BS, Isacsson SO, Tejler L. Social network, social support and acute chest complaints among young and middle-aged patients in an Emergency Department-a case control study. Soc Sci Med. 1991;33(3):257-67. http://dx.doi.org/10.1016/0277-9536(91)90359-K. PMid:1925689.

17. Gobbi BC, Cunha EP, Brito MJ, Senger I. Politizando o conceito de redes organizacionais: uma reflexão teórica da governança como jogo de poder. Cadernos Ebape. 2005;3(1):1-16. http://dx.doi.org/10.1590/ S1679-39512005000100004. 
18. Silva LA, Casotti CA, Chaves SC. A produção científica brasileira sobre a Estratégia Saúde da Família e a mudança no modelo de atenção. Cien Saude Colet. 2013;18(1):221-32. http://dx.doi.org/10.1590/S141381232013000100023 . PMid:23338512.

19. Campos PCM, Gomide M. O Programa Nacional de Controle do Tabagismo (PNCT) na perspectiva social: a análise de redes, capital e apoio social. Cad Saude Colet. 2015;23(4):436-44. http://dx.doi. org/10.1590/1414-462X201500040241.

20. Portugal S. Contributos para uma discussão do conceito de redes na teoria sociológica. Oficina CES. 271;2007:1-36.

21. Organização Mundial da Saúde. CID-10 Classificação Estatística Internacional de Doenças e Problemas Relacionados à Saúde. 10. ed. São Paulo: Universidade de São Paulo; 1997. Vol. 2.

22. West R. Assessment of dependence and motivation to stop smoking. BMJ. 2004;328(7435):338-9. http:// dx.doi.org/10.1136/bmj.328.7435.338. PMid:14764501.

23. Speck VR. La intervención en red social: Las terapias de red, teoria y desarrollo. In: Elkaim M. Em La práctica de la terapia de red. Barcelona: Gedisa; 1989. p. 20-39. 